\title{
AMPELOGRAPHIC CHARACTERIZATION OF TURKISH INDIGENOUS GRAPE ACCESSIONS AND EUROPEAN CULTIVARS (Vitis vinifera $\mathbf{L}$.)
}

\author{
Burcak ISCI $^{1, *}$ Ahmet ALTINDISLI ${ }^{1 \oplus}$ \\ 'Department of Horticulture, Agriculture Faculty, Ege University, 35100, Izmir/Turkey \\ "Corresponding Author: burcak.isci@ege.edu.tr
}

\begin{abstract}
A total of 35 grape accessions and 3 reference cultivars were used to investigate the genetic polymorphism and relationships among Turkey and other European grape accessions by ampelographic characterization. Total of 74 ampelographic characteristics were identified for 38 genotypes. Ampelographic data were collected two vegetation periods. The characteristics of the vines were defined and measured according to OIV descriptors. In this study, three synonym varieties (100\% similarity) were identified: 'Cabernet Sauvignon' and 'Cabernet Franch'; standard grape variety 'Merlot' and no.12 genotype (Merlot), and 'Sèmillion' analyzed as a standard grape variety and no. 1 'Sèmillion' genotype. Based on similarity rate of grape varieties, highest similarity ratios were found between 'Yuvarlak Razak1-Siyah Gemre' with 93\%, 'Yuvarlak Razakı-Siyah Gemre' and 'Şika' with 91\%, 'Moiseylative-Hafızali' with 90\% and 'Kırmızı Şam-Pembe Gemre' genotypes with 89\%. In principal component analyses graph, 'Müşküle', 'Buca Razakı', 'Moiseylative', 'Kırmızı Şam', 'Cardinal', 'Yuvarlak Razakı', 'Hafızali', 'Siyah Gemre' and 'Şika' were grouped together.
\end{abstract}

Keywords: Vitis vinifera L., Ampelography, Characterization, Grape Accessions

Received: 23.10.2017 Accepted: 09.11.2017 Published: 20.12.2017

\section{INTRODUCTION}

Anatolia has a long history of viticulture and a wide diversity of grape cultivars. Grapevines (Vitis vinifera L.) are one of the oldest domesticated crop plants and economically the most important cultivated fruit crops in the world. Turkey, one of the countries where Vitis vinifera L. was first cultivated, has a rich grapevine gene potential. According to Oraman and Ağaoğlu, 1969 [1], Turkey has a history of viticulture dating back to 3500 B.C. Grapes have an important place among agricultural products as table grapes, wine grapes, raisin and with their various local uses.

Ampelographic studies have been undertaken for many years to present the grapevine gene potential in Turkey. As in many parts of the world, heterozygotic hereditary structure of the grapevine has resulted in the generation of a wide variety, type and species in Turkey, a country regarded as the homeland of viticulture for Vitis vinifera $\mathrm{L}$. Investigation of genetic relationship is very import for germplasm conservation, evaluation and utilization for future grape breeding programs considering the present need of cultivar improvement. The objectives of the present study are to investigate the genetic relationships among Turkey grape accessions which include main local grape varieties in Turkey and some European cultivars were investigated for ampelographic observations of a total of 38 grape accessions (Vitis vinifera L.) including the 35 grape accessions and 3 reference cultivars. Scientific studies undertaken to identify the grapevine gene potential in Turkey and to prepare a catalog for this potential to validate it in international contexts are crucial. 


\section{MATERIALS AND METHODS}

\section{Plant Material}

Thirty five grapevine cultivars (Vitis vinifera L.) were analyzed to determine their ampelographic relationships. The representative vines of cultivars were grown in implementation area at Horticulture Department, Agriculture Faculty, Ege University. The locations of the vineyards: $38^{0} 27^{\prime} 32^{\prime \prime} \mathrm{N}, 27^{0} 1321^{\prime \prime} \mathrm{E}$. Nine vines per cultivar were selected for study. The vines were 12 years old and cultivated under the same growing conditions using rootstock 41B (Vinifera $\mathrm{x}$ Berlandieri) with the spaces $2,5 \times 3 \mathrm{~m}$.

Three reference cultivars, Vitis vinifera L. cv. 'Cabernet Sauvignon', Vitis vinifera L. cv. 'Merlot' and Vitis vinifera L. cv. 'Sémillon' were considered as reference cultivars, as they were recently characterized in detail using SSR primers of core set, VVS2, VVMD5, VVMD7, VVMD27, VrZAG62 and VrZAG79 [2], they were grown at Manisa Viticulture Research and implementation area (Manisa/Turkey). Basic ampelographic characteristics of grape varieties and reference cultivars used in this study is listed in Table 1.

\section{Ampelografic Evaluation}

Ampelographic characterization of 38 grapevine genotypes was conducted using the descriptions in the Descriptors for Grapevine (Vitis spp.) (GENRES 081 1997) and the Office International de la Vigne et du Vin (OIV) Descriptor List for Grape Varieties and Vitis species $[3,4]$. Descriptors used in this study and their OIV-IPGRI codes are presented in Table 2. In total, 75 different descriptors were used.

Ampelographic observations were carried out during two consecutive vegetation periods. The characteristics of the vines were defined and measured according to OIV descriptors. The characters of representing vines were investigated/measured following the specifications of vine growth stages indicated by OIV. The shoot tips were investigated when they were approximately 10 to $30 \mathrm{~cm}$ in height, and the first four distal leaves of young leaves were evaluated.

Mature leaf descriptions were obtained between berry set and beginning of berry maturity and were conducted on leaves above the cluster within the middle of the shoot. The clusters were measured at maturity and berry characteristics were obtained from ripe berries located in the middle of the bunch.

On average, ten canes per variety were analyzed after leaf fall. The mean values obtained over two years were transformed to numerical scales according to international descriptors. The resulting raw data were analyzed in NTSYSpc 2.0 software [5] using a distance matrix. The clustering dendrogram was based on the unweighted pair group of the arithmetic mean (UPGMA) [3]. A principal component analysis (PCA) graph was also constructed.

\section{Ampelografic Analysis Evaluation}

For analysis, dendrograms for genotypes according to UPGMA (Unweighted pair-group method arithmetic average) grouping were obtained by using NTSYS-version 2.0 (Numerical Taxonomy and Multivariate Analysis System) [5] statistical package program.

\section{RESULTS AND DISCUSSION}

Ampelografic studies are utilized to identify existing and new breeds in all the countries where viticulture is common. Identification of the ampelografic characteristics of grapevine accessions and varieties is crucial for identification and classification. It is important to know grape accession qualities to determine the best adapted varieties and to plan breeding work. In this study, ampelografic observations were carried out by investigating 73 features in 38 grape accessions based on "Descriptors for Grape".

According to ampelografic observation results presented in Table 2, all grape accessions in the study were identified as Vitis vinifera $\mathrm{L}$. since their shoot types (OIV 001) were "open=7" and sequencing of tendrils (OIV 16) were "discontinuous $=1$ " i.e. " 2 or less".

Density of anthocyan on the tip of shoots (OIV 003 ) was not observed in 'Müşküle', 'Moiseylative', 'Pek Üzümü', 'İtalia', 'Siyah Gemre', 'Colombard', 'Abiguş' and 'Kırmızı Şam' grape varieties $(0=$ absent $)$, it was found to be medium in 'Cabernet Sauvignon' and 'Cabernet Franc' 'Şam' grape varieties $(5=$ medium $)$ and it was weak $(3=$ weak $)$ in other grape varieties.

Examination of OIV 244 descriptor (seed: tranversal ridges on side) shows that lack of tranversal ridges on side of the seed $(a b s e n t=0)$ validates the variety as belonging to Vitis vinifera L. 
Examination of flower types (OIV 151) shows that all grape acccessions had hermaphrodite (hermaphrodite $=3$ ) structure.

It is very important to examine mature leaf characteristics in ampelografic definitions. Based on "Mature leaf: number of lobes" (OIV 068) descriptor, 'Yuvarlak Razakı' and 'Çeşme Pembesi' grape varieties were found to have 3 lobes "three $=2$ " while the others had five lobes five $=3$ " (Table 2). Definitions relevant to mature leaves have been generally approved as powerful way of identifying grapevine genotypes [6, 7]. In a similar studies, Ecevit and Kelen 1999, [8] and Ateş et al., 2011 [9] also reported the leaves with five lobes as a major type among some Turkish grapes.

All grape accessions in this study were determined to be "both sides convex $=3$ " based on mature leaf: shape of teeth (OIV 076) descriptor. Differences were observed between varieties in terms of "Mature leaf: length" (OIV 066) and "mature leaf: shape of base of petiole sinus" (OIV 080) descriptors. 'Tarsus Pembesi' and 'Granache' varieties were in "short=3" group in terms of leaf size whereas other varieties were in "very short=1" group.

According to "Mature leaf: general shape of petiole sinus" (OIV 079) descriptor, 'Italia', 'Sémillon' and 'Çeşme Pembesi' grape varieties were found to be "wide open=2" and the others were identified to be "open=3".

Differences were identified among grape accessions in terms of cluster characteristics. Three separate groups were identified among the varieties with "Bunch: length" (OIV 203) descriptor. The majority of the grape varieties were included in "very short $(<11 \mathrm{~cm})$ " classification group while 'Kırmızı Şam' and 'Moiseylative' varieties were in "long (24-26 cm)" group. 'Öküzgözü', 'Kozak Gemresi', 'Çeşme Pembesi', 'Cardinal', 'Ohannes', 'Siyah Gemre' and 'Yuvarlak Razak1' grape varieties were in "short (14-16 cm)" group. As stated by Marasali, 1986 [10] and Demir, 1987 [11], cluster lengths can differ based on whether the variety is situated in its own ecology or its adaptation to the ecology it is situated in.

Evaluation of results obtained from "Berry: uniformity of size" (OIV 222) descriptor shows differences among varieties and it was found that berries were generally uniform in clusters.

According to "Berry: shape" based on OIV 223 descriptor: The majority of the grape varieties were found to have round, while 'Şika' and 'Çeşme
Pembesi' ovate, 'Delbele' obovate, 'Öküzgözü', 'Sémillon' and 'Alicante Boushet' slightly flat, 'Pek Üzümü', 'Hafizali', 'Conlonbart' and 'Moiseylative' obtuse-ovate, 'Cinsaut' and 'Buca Razakısı' were found to have long eliptic shape.

In terms of "Berry: classification of flavor" (OIV 237) descriptor, 'Cardinal', 'Merlot' and 'Harsleleh' grape varieties were found to have unique flavors and were included in "little flavor=2" classification group.

The UPGMA dendrogram, constructed on the basis of ampelographic scoring (0 to 9) using a distance matrix, is shown in Figure 1. Average similarity ratio of genotypes is $84 \%$. Genotypes have shown various types of branching in ampelographic dendrogram. In the first differentiation of the dendrogram, 'Abiguş' grape cultivar generated a different group from other varieties. In the second differentiation of the dendrogram, 'Öküzgözü', 'Grenache Noir', 'Italia', 'Kozak Gemresi', 'Kırmızı Şam', 'Pembe Gemre', 'Yuvarlak Razakı', 'Siyah Gemre', 'Şika', 'Cardinal', 'Moiseylative', 'Hafizali', 'Buca Razak1', 'Pek Üzümü', 'Çeşme Pembesi', 'Mahrabaş1', 'Tarsus Pembesi', 'Beyaz Şam', 'Ohannes', 'Müşküle' genotypes created sub groups and were distributed along the dendrogram. In the third differentiation point, some groups of two or three were found to be generated from the three reference varieties and 14 genotypes.

As can be seen in the dendrogram, three synonym varieties (100\% similarity) were identified: 'Cabernet' and 'Cabernet Franch', standard grape variety 'Merlot' and no.12 genotype (Merlot) and 'Sèmillion' analyzed as a standard grape variety and no. 1 'Sèmillion' genotype.

Based on similarity rate of grape varieties, highest similarity ratios were found between 'Yuvarlak Razak1-Siyah Gemre' with 93\%, 'Yuvarlak Razakı-Siyah Gemre' and 'Şika' with 91\%, 'Moiseylative-Hafizali' with $90 \%$ and 'Kırmızı Şam-Pembe Gemre' genotypes with $89 \%$. As seen in principal component analyses graph, 'Müşküle', 'Buca Razak1', 'Moiseylative', 'Kırmızı Şam', 'Cardinal', 'Yuvarlak Razakı', 'Hafizali', 'Siyah Gemre' and 'Şika' were grouped together. No links were found with being local or universal varieties (Figure 1, Figure 2).

Prevalence of homonym and synonym groups were also presented in studies implemented with Turkish grape accessions $[12,13,14,15,16,17$, $18,19,20,21]$. Development of viticulture in 
Turkey will be possible when scientific studies that will allow the identification and preservation of our rich grapevine gene potential and provide resources for new breeding studies will be given the required importance. It is imperative to test the ampelographic characteristics of grape varieties and their relationships at genetic levels through modern methods that have international levels of reliability in order to conserve grape varieties in Turkey and select high quality varieties from among them. It is crucial to identify the genotypes via exact identification since some genotypes are names the same. In addition, the present study provided particular knowledge on some autochthonous grapevine cultivars, most of which are today on the verge of extinction. This study would therefore help to prevent disappearing local cultivars and to preserve such germplasm collection for the future studies. It is hoped that this study will enlighten similar studies in the field in the future.

\section{ACKNOWLEDGMENTS}

This work was financially supported by Ege University Scientific Research Fund (project number: 06-ZRF-007).

\section{REFERENCES}

[1] Oraman, MN. and Agaoglu, YS. (1969). Some characteristics of Turkey's viticulture and the composition of its districts in viticulture. Ankara University Agriculture Faculty Yearbook, Ankara.

[2] İşçi, B. and Dilli, Y. (2014). Ege Bölgesi Yerel Asma Çeşitlerinin (Vitis vinifera L.) basit tekrar dizileri (SSRs) ile karakterizasyonu. Tarım Bilimleri Dergisi, 21 (4), 538-545.

[3] OIV (2001). Second Edition of the OIV Descriptor list for grape varieties and Vitis Species Website: http://www.oiv.int

[4] Anonymous (2013). GENRES Informationssystem Genetische Ressourcen, http://www.genres.de/vitis/

[5] Rohlf, F.J. (2000). Phylogenetic models and reticulations. J. Classif., 17, 185-189.

[6] Ortiz, J. M., Martin, J. P. Borrego,J. Chavez, J. Rodriguez, I. Munoz, G. and Cabello, F. (2004). Molecular and morphological characterization of a Vitis gene bank for the establishment of a base collection. Genetic Research and Crop Evaluation, 51: 403-409.

[7] Santiago, J. L., Boso, S. Gago, P. AlonsoVillaverde, V. and Martinez, M.C. (2007).
Molecular and ampelographic characterization of Vitis vinifera L. 'Albarino', 'Savagnin Blanc' and 'Caino Blanco' shows that they are different cultivars. Spanish Journal of Agricultural Research, 5:333-340.

[8] Ecevit, F. M. and Kelen, M. (1999). Determination of ampelographical characters of grape cultivars grown in Isparta (Atabey). Turkish Journal of Agriculture and Forestry, 23:511-518.

[9] Ateş, F. Çoban, H. Kara, Z. and Sabır, A. (2011). Ampelographic characterization of some grape cultivars (Vitis vinifera L.) grown in South-western region of Turkey. Bulgarian Journal of Agricultural Science, 17 (3), 314324.

[10] Marasal1, B. (1986). Determination of the ampelographic characters of some native standard grape varieties grown in Ankara conditions. Master Thesis, Ankara University. Graduate School of Natural and Sciences, Ankara.

[11] Demir, I. (1987). Studies on identification of ampelographic characteristics of foreign origin grape varieties cultivated in Ankara. Master Thesis, Ankara University. Graduate School of Natural and Sciences, Ankara.

[12]Ergül, A., Kazan, K., Aras, S., Çevik, V., Çelik, H. and Söylemezoğlu, G. (2006). AFLP analysis of genetic variation within the two economically important Anatolian grapevine (Vitis vinifera L.) varietal groups. Genoma, 49: 467-475.

[13] Karaağaç, E. (2006). Molecular analysis of Gaziantep province grapevine gene potential through SSR Markers. Doctora Thesis, Ankara University. Graduate School of Natural and Sciences, Ankara.

[14] Vouillamoz, J.F., Mcgovern, P.E., Ergül, A., Soylemezoglu, G., Tevzadze, G., Meredith, C.P. and Grando, M.S. (2006). Genetic characterization and relationships of traditional grape cultivars from Transcaucasia and Anatolia. Plant Genetic Resources: Characterization \& Utilization, 4(2), 144158.

[15] Selli, F., M. Bakır, G. İnan, H. Aygün, Y. Boz, A. S. Yaşasın, C. Ozer, B. Akman, Soylemezoğlu, G., Kazan, K. and Ergül, A. (2007). Simple Sequence Repeat-Based assesment of genetic diversity in "Dimrit" and "Gemre" grapevine accessions from Turkey. Vitis, 46(4), 182-187.

[16] Yildirım, N. (2008). Characterization of Kara (Grape) Groups based on SSR(Simple Sequence Repeat) markers and genetic relations of these cultivars and national grapevine Germplasms. Thesis, Ankara 
Üniversity, Biotechnology Enstitues.

[17] Shidfar, M. (2008). Genetic characterization of Eskisehir and Kayseri province grapevine gene sources based on SSRs (Simple Sequence Repeats). Master Thesis, Ankara University. Graduate School of Natural and Sciences, Ankara.

[18] Hizarc1, Y., Ercişli, S., Yüksel, C. and Ergül, A. (2012). Genetic characterization and relatedness among autochthonous grapevine cultivars from Northeast Turkey by Simple Sequence Repeats (SSR). Journal of Applied Botany and Food Quality, 85, 224-228.

[19] Dangl, G.S., Mendum, M.L., Prins, B.H., Walker, A.M., Meredith, C.P. and Simon C.J. (2001). Simple sequence repeat analysis of a clonally propagated species: A tool for managing a grape germplasm collection.
Genome, 44, 432-438.

[20] Martın, J.P., Borrego, J., Cabello, F. and Ortız, J.M. (2003). Characterization of the Spanish diversity grapevine cultivars using sequencetagged microsatellite site markers. Genome, 46, 1-9.

[21] This, P., Jung, A., Boccacc1, P., Borrego, J., Botta, R., Costantını, L., Crespan, M., Dangl, G.S., Eisenheld, C., Ferreira Monteiro, F., Grando, M.S., Ibanez, J., Lacombe, T., Laucou, V., Magalhaes, N., Meredith, C.P., Milanı, N., Peterlunger, E., Regner, F., Zulını, L. and Maul, E. (2004). Development of a standard set of microsatellite reference alleles for identification of grape cultivars. Theoretical and Applied Genetics, 109, 1448-1458.

Table 1. Basic ampelographic characteristics of the grape cultivars used in this study.

\begin{tabular}{|c|c|c|c|c|c|}
\hline Cultivar & Cluster Form & Berry Form & Berry Colour & Flavor & Seed \\
\hline CabernetSauvignon & Very dense & Round & Blue-black & Little flavour & Present \\
\hline Merlot & Very dense & Round & Blue-black & Little aromatic & Present \\
\hline Sémillon & Dense & Round & Greenyellow & Neutral & Present \\
\hline Sémillon & Dense & Round & Greenyellow & Neutral & Present \\
\hline Marsleleh & Dense & Long elliptic & Dark redviolet & Neutral & Present \\
\hline Conlonbart & Medium & Obtuseovate & Greenyellow & Neutral & Present \\
\hline Grenache Noir & Dense & Round & Dark redviolet & Neutral & Present \\
\hline Cinsaut & Dense & Long elliptic & Blue-black & Neutral & Present \\
\hline Cabernet Fanc & Dense & Round & Blue-black & Neutral & Present \\
\hline CabernetSauvignon & Very dense & Round & Blue-black & Little flavour & Present \\
\hline Papazkarası & Very dense & Round & Red & Little flavour & Absent \\
\hline Öküzgözü & Loose & Slightly flat & Rose & Little flavour & Present \\
\hline Petit Syrah & Dense & Round & Blue-black & Neutral & Present \\
\hline Foça Karası & Very dense & Round & Blue-black & Neutral & Present \\
\hline Merlot & Very dense & Round & Blue-black & Little aromatic & Present \\
\hline Alicante Boushet & Very dense & Slightly flat & Blue-black & Little flavour & Present \\
\hline Delbele & Medium & Obovate & Blue-black & Neural & Present \\
\hline Grenache & Medium & Round & Blue-black & Little flavour & Present \\
\hline Malbee & Dense & Round & Blue-black & Neutral & Present \\
\hline Çeşme Pembesi & Loose & Ovate & Rose & Neutral & Present \\
\hline Kozak Gemresi & Loose & Round & Red & Neutral & Present \\
\hline Abiguş & Loose & Round & Red & Neutral & Present \\
\hline Kır mızı Şam & Very loose & Round & Red & Little flavour & Present \\
\hline Mahrabaşı & Loose & Round & Dark redviolet & Neutral & Present \\
\hline Yuvarlak Razakı & Loose & Round & Greenyellow & Neutral & Present \\
\hline Siyah Gemre & Loose & Round & Red & Neutral & Present \\
\hline Pembe Gemre & Loose & Round & Rose & Neutral & Present \\
\hline Cardinal & Loose & Round & Blue-black & Little aromatic & Present \\
\hline Beyaz Şam & Loose & Round & Greenyellow & Neutral & Present \\
\hline Italia & Loose & Round & Red & Little flavour & Present \\
\hline Ohannes & Loose & Round & Greenyellow & Little flavour & Present \\
\hline Pek Üzümü & Medium & Obtuseovate & Blue-black & Little flavour & Present \\
\hline Şika & Loose & Ovate & Dark redviolet & Neutral & Present \\
\hline Müşküle & Loose & Round & Dark redviolet & Neutral & Present \\
\hline Moiseylative & Medium & Obtuseovate & Blue-black & Little flavour & Present \\
\hline Buca Razakısı & Loose & Long elliptic & Greenyellow & Little flavour & Present \\
\hline Tarsus Pembesi & Loose & Round & Rose & Neutral & Present \\
\hline Hafizali & Loose & Obtuseovate & Blue-black & Neutral & Absent \\
\hline
\end{tabular}


Table 2. Ampelographic characteristics of grape cultivars used in this study.

\begin{tabular}{|c|c|c|c|c|c|c|c|}
\hline $\begin{array}{l}\text { OIV } \\
\text { Cod } \\
\text { Number }\end{array}$ & Tarsus Pembesi ${ }^{\star}$ & Buca Razakısı & Moiseylative & Müşküle & Hafızali & Şika & Pek Üzümü \\
\hline 001 & Open & Half-open & Half-open & Half-open & Half-open & Half-open & Half-open \\
\hline 003 & Very weak & Very weak & Absent & Absent & Very weak & Very weak & Absent \\
\hline 004 & Sparse & Very sparse & Sparse & Very sparse & Very sparse & Very sparse & Very sparse \\
\hline 005 & None & None & None & None & None & None & None \\
\hline 006 & Semierect & Semierect & Semierect & Semierect & Semierect & Horizontal & Semierect \\
\hline 007 & Green & G with r.s & G with r.s & G with r.s & Green & Green & G with r.s \\
\hline 008 & G with r.s & G with r.s & G with r.s & G with r.s & Green & Green & G with r.s \\
\hline 009 & G with r.s & Green & G with r.s & G with r.s & G with r.s & G with r.s & G with r.s \\
\hline 010 & G with r.s & G with r.s & G with r.s & G with r.s & G with r.s & G with r.s & G with r.s \\
\hline 011 & None & None & None & None & None & None & None \\
\hline 012 & None & None & None & None & None & None & None \\
\hline 013 & Very spase & None & None & None & None & None & None \\
\hline 014 & None & None & None & None & None & None & None \\
\hline 015 & Very weak & Absent & Absent & Very weak & Absent & Very weak & Weak \\
\hline 016 & Dis. cont. & Dis. cont. & Dis. cont. & Dis. cont. & Dis. cont. & Dis. cont. & Dis. cont. \\
\hline 017 & Short & Short & Very short & Short & Very short & Short & Very short \\
\hline 051 & $\begin{array}{l}\text { Green with } \\
\text { b.s. }\end{array}$ & $\begin{array}{l}\text { Green with } \\
\text { b.s. }\end{array}$ & $\begin{array}{l}\text { Green with } \\
\text { b.s. }\end{array}$ & $\begin{array}{l}\text { Green with } \\
\text { b.s. }\end{array}$ & $\begin{array}{l}\text { Green with } \\
\text { b.s. }\end{array}$ & $\begin{array}{l}\text { Green with } \\
\text { b.s. }\end{array}$ & $\begin{array}{l}\text { Green with } \\
\text { b.s. }\end{array}$ \\
\hline 052 & None & None & Very weak & Weak & None & None & None \\
\hline 053 & Dense & None & None & None & Very sparse & Very sparse & Very sparse \\
\hline 054 & Sparse & None & None & None & None & None & None \\
\hline 055 & Sparse & None & None & None & None & None & None \\
\hline 056 & None & Very sparse & Very sparse & None & None & Very sparse & None \\
\hline 065 & Small & Very small & Very small & Very small & Very small & Very small & Very small \\
\hline 066 & Short & Short & Very short & Very short & Short & Short & Short \\
\hline 067 & Pentagonal & Pentagonal & Pentagonal & Pentagonal & Pentagonal & Pentagonal & Pentagonal \\
\hline 068 & Five & Five & Five & Five & Five & Five & Five \\
\hline 069 & Medium green & Medium green & Dark green & Pale green & Dark green & Dark green & Dark green \\
\hline 070 & Absent & Absent & Very weak & Absent & Absent & Absent & Absent \\
\hline 071 & Absent & Absent & Absent & Absent & Absent & Absent & Absent \\
\hline 076 & B. s. straight & B. s. straight & B. s. straight & B. s. straight & B. s. straight & B. s. straight & B. s. straight \\
\hline 077 & Very long & Long & Long & Long & Medium & Very long & Long \\
\hline 078 & Long & Long & Long & Long & Long & Long & Medium \\
\hline 079 & Wide open & Open & Open & Open & Open & Wide open & Open \\
\hline 080 & U shape & V shape & V shape & V shape & U shape & U shape & V shape \\
\hline 081 & None & None & None & None & None & None & None \\
\hline 082 & Closed & Open & L. s. overlap. & L. s. overlap. & Open & Open & Open \\
\hline 083 & V shape & V shape & V shape & U shape & V shape & U shape & V shape \\
\hline 084 & None & None & None & None & None & None & None \\
\hline 085 & None & None & None & None & None & None & None \\
\hline 086 & None & None & Very sparse & None & None & None & Spase \\
\hline 087 & None & Very sparse & None & None & None & None & None \\
\hline 088 & Absent & Absent & Absent & Absent & Absent & Absent & Absent \\
\hline 089 & Absent & Absent & Absent & Absent & Absent & Absent & Absent \\
\hline 090 & None & None & None & None & None & None & None \\
\hline 091 & None & None & None & None & None & None & None \\
\hline 092 & Very short & Very short & Very short & Very short & Very short & Very short & Very short \\
\hline 093 & Shorter & Shorter & Shorter & Longer & Shorter & Shorter & Shorter \\
\hline 151 & Hermaphrodite & Hermaphrodite & Hermaphrodite & Hermaphrodite & Hermaphrodite & Hermaphrdite & Hermaphrodite \\
\hline 153 & $\begin{array}{l}1.1 \text { to } 2 \\
\text { inflorescenses }\end{array}$ & $\begin{array}{l}1.1 \text { to } 2 \\
\text { inflorescenses }\end{array}$ & $\begin{array}{l}1.1 \text { to } 2 \\
\text { inflorescenses }\end{array}$ & $\begin{array}{l}1.1 \text { to } 2 \\
\text { inflorescenses }\end{array}$ & $\begin{array}{l}1.1 \text { to } 2 \\
\text { inflorescenses }\end{array}$ & $\begin{array}{l}1.1 \text { to } 2 \\
\text { inflorescenses }\end{array}$ & $\begin{array}{l}1.1 \text { to } 2 \\
\text { inflorescenses }\end{array}$ \\
\hline
\end{tabular}


Table 2. Ampelographic characteristics of grape cultivars used in this study (continued).

\begin{tabular}{|l|l|l|l|l|l|l|l|}
\hline $\begin{array}{l}\text { OIV } \\
\text { Cod } \\
\text { Number }\end{array}$ & Tarsus Pembesi & Buca Razakıs & Moiseylative & \multicolumn{1}{|c|}{ Müşküle } & Hafızali & Şika & Pek Üzümü \\
\hline 154 & & & & & & & \\
\hline 203 & & Long & Very long & Long & Medium & Very long & Medium \\
\hline 204 & & Short & Long & Short & Short & Short & Medium \\
\hline 205 & & Loose & Medium & Loose & Loose & Loose & Medium \\
\hline 206 & & Very few & Medium & Few & Very few & Few & Few \\
\hline 207 & & Very long & Very long & Very long & Very long & Very long & Very long \\
\hline 221 & & Weak & Medium & Strong & Weak & Weak & Weak \\
\hline 222 & & Long & Long & Medium & Long & Medium & Medium \\
\hline 223 & & Not uniform & Not uniform & Not uniform & Not uniform & Uniform & Uniform \\
\hline 224 & & Long elliptic & Obtuseovate & Round & Obtuseovate & Ovate & Obtuseovate \\
\hline 225 & & Not circular & Not circular & Circular & Circular & Circular & Circular \\
\hline 226 & & Greenyellow & Blue-black & Dark redviolet & Blue-black & Dark rect & Blue-black \\
\hline $230-231$ & & & & & & violet & \\
\hline 233 & & Not uniform & Not uniform & Not uniform & Not uniform & Not uniform & Not uniform \\
\hline 237 & & Not coloured & Not coloured & Not coloured & Not coloured & Not coloured & Not coloured \\
\hline 238 & & Medium & Medium & Medium & Medium & Little & Medium \\
\hline 239 & & Little flavour & Little flavour & Neutral & Neutral & Neutral & Little flavour \\
\hline 241 & & Medium & Short & Short & Short & Short & Short \\
\hline 301 & Late & Medim & Medim & Easy & Medim & Medim & Medim \\
\hline 305 & Medium & Present & Present & Present & Absent & Present & Present \\
\hline 306 & Yellow & Ledium & Late & Medium & Late & Medium & Very early \\
\hline
\end{tabular}


Table 2. Ampelographic characteristics of grape cultivars used in this study (continued).

\begin{tabular}{|c|c|c|c|c|c|c|c|}
\hline $\begin{array}{l}\text { OIV } \\
\text { Cod } \\
\text { Number }\end{array}$ & Beyaz Şam* & Italia & Ohannes & Cardinal & $\begin{array}{l}\text { Pembe } \\
\text { Gemre* }\end{array}$ & Siyah Gemre & $\begin{array}{c}\text { Yuvarlak } \\
\text { Razakı }\end{array}$ \\
\hline 001 & Half-open & Half-open & Half-open & Half-open & Half-open & Half-open & Half-open \\
\hline 003 & Weak & Absent & Very weak & Very weak & Very weak & Absent & Very weak \\
\hline 004 & Medium & Dense & Sparse & Very sparse & Medium & Very sparse & Very sparse \\
\hline 005 & None & None & None & None & None & None & None \\
\hline 006 & Horizontal & Semierect & Horizontal & Semierect & Semierect & Semierect & Semierect \\
\hline 007 & G with r.s & G with r.s & Green & G with r.s & G with r.s & G with r.s & Green \\
\hline 008 & G with r.s & Green & G with r.s & G with r.s & G with r.s & G with r.s & Green \\
\hline 009 & G with r.s & G with r.s & G with r.s & G with r.s & G with r.s & G with r.s & Green \\
\hline 010 & G with r.s & Green & G with r.s & G with r.s & G with r.s & G with r.s & Green \\
\hline 011 & None & None & None & None & None & None & None \\
\hline 012 & None & None & None & None & None & None & None \\
\hline 013 & None & None & None & None & None & None & None \\
\hline 014 & None & None & None & None & None & None & None \\
\hline 015 & Absent & Absent & Weak & Very weak & Absent & Very weak & Absent \\
\hline 016 & Dis. cont. & Dis. cont. & Dis. cont. & Dis. cont. & Dis. cont. & Dis. cont. & Dis. cont. \\
\hline 017 & Very short & Very short & Short & Short & Very short & Short & Very short \\
\hline 051 & $\begin{array}{l}\text { Green with } \\
\text { b.s. }\end{array}$ & Green & Green with b.s. & $\begin{array}{l}\text { Green with } \\
\text { b.s. }\end{array}$ & $\begin{array}{l}\text { Green with } \\
\text { b.s. }\end{array}$ & $\begin{array}{l}\text { Green with } \\
\text { b.s. }\end{array}$ & $\begin{array}{l}\text { Green with } \\
\text { b.s. }\end{array}$ \\
\hline 052 & Very weak & None & None & None & None & None & None \\
\hline 053 & Very sparse & Medium & Sparse & Sparse & Medium & Very sparse & Çok seyrek \\
\hline 054 & None & None & None & None & None & None & None \\
\hline 055 & Very sparse & Sparse & None & None & None & None & None \\
\hline 056 & Sparse & Sparse & Very sparse & None & Very sparse & None & None \\
\hline 065 & Very small & Very small & Very small & Very small & Very small & Very small & Very small \\
\hline 066 & Short & Very short & Very short & Short & Short & Short & Short \\
\hline 067 & Pentagonal & Pentagonal & Pentagonal & Pentagonal & Pentagonal & Pentagonal & Pentagonal \\
\hline 068 & Five & Five & Five & Five & Five & Five & Three \\
\hline 069 & Medium green & Pale green & Pale green & Dark green & Medium green & Dark green & Medium green \\
\hline 070 & Absent & Absent & Absent & Absent & Absent & Absent & Absent \\
\hline 071 & Absent & Absent & Absent & Absent & Absent & Absent & Absent \\
\hline 076 & B. s. straight & B. s. straight & B. s. straight & B. s. straight & B. s. straight & B. s. straight & B. s. straight \\
\hline 077 & Long & Short & Long & Medium & Very long & Medium & Long \\
\hline 078 & Very long & Short & Long & Long & Very long & Long & Long \\
\hline 079 & Open & Wide open & Open & Open & Open & Open & Open \\
\hline 080 & V shape & V shape & V shape & U shape & V shape & U shape & U shape \\
\hline 081 & None & None & None & None & None & None & None \\
\hline 082 & Open & Open & Open & L. s. overlap. & Open & Open & Open \\
\hline 083 & U shape & U shape & V shape & U shape & V shape & V shape & U shape \\
\hline 084 & None & None & None & None & None & None & None \\
\hline 085 & None & Very weak & None & None & None & None & None \\
\hline 086 & Very sparse & Very sparse & None & None & Very sparse & Very sparse & Very sparse \\
\hline 087 & None & None & None & None & None & None & None \\
\hline 088 & Absent & Absent & Absent & Absent & Absent & Absent & Absent \\
\hline 089 & Absent & Absent & Absent & Absent & Absent & Absent & Absent \\
\hline 089 & Absent & Absent & Absent & Absent & Absent & Absent & Absent \\
\hline 090 & None & None & None & None & None & None & None \\
\hline 091 & None & None & None & None & None & None & None \\
\hline 092 & Very short & Very short & Very short & Very short & Very short & Very short & Very short \\
\hline 093 & Shorter & Shorter & $\begin{array}{l}\text { Very much } \\
\text { shorter }\end{array}$ & Shorter & Shorter & Shorter & Shorter \\
\hline 151 & Hermaphrodite & Hermaphrodite & Hermaphrodite & Hermaphrodite & Hermaphrodite & Hermaphrodite & Hermaphrodite \\
\hline 153 & $\begin{array}{l}1.1 \text { to } 2 \\
\text { inflorescenses }\end{array}$ & $\begin{array}{l}1.1 \text { to } 2 \\
\text { inflorescenses }\end{array}$ & $\begin{array}{l}1.1 \text { to } 3 \\
\text { inflorescenses }\end{array}$ & $\begin{array}{l}1.1 \text { to } 2 \\
\text { inflorescenses }\end{array}$ & $\begin{array}{l}1.1 \text { to } 2 \\
\text { inflorescenses }\end{array}$ & $\begin{array}{l}1.1 \text { to } 2 \\
\text { inflorescenses }\end{array}$ & $\begin{array}{l}1.1 \text { to } 2 \\
\text { inflorescenses }\end{array}$ \\
\hline
\end{tabular}


Table 2. Ampelographic characteristics of grape cultivars used in this study (continued).

\begin{tabular}{|c|c|c|c|c|c|c|c|}
\hline $\begin{array}{l}\text { OIV } \\
\text { Cod } \\
\text { Number }\end{array}$ & Beyaz Şam* & Italia & Ohannes & Cardinal & $\begin{array}{l}\text { Pembe } \\
\text { Gemre* }\end{array}$ & $\begin{array}{l}\text { Siyah } \\
\text { Gemre }\end{array}$ & $\begin{array}{c}\text { Yuvarlak } \\
\text { Razakı }\end{array}$ \\
\hline 154 & Short & Short & Short & Very long & Long & Long & Very long \\
\hline 203 & & Very short & Short & Short & & Short & Short \\
\hline 204 & & Loose & Loose & Loose & & Loose & Loose \\
\hline 205 & & Very few & Few & Few & & Very few & Very few \\
\hline 206 & & Very long & Very long & Very long & & Very long & Very long \\
\hline 207 & & Strong & Medium & Weak & & Weak & Weak \\
\hline 221 & & Long & Medium & Long & & Medium & Medium \\
\hline 222 & & Uniform & Not uniform & Not uniform & & $\begin{array}{l}\text { Not } \\
\text { uniform }\end{array}$ & Not uniform \\
\hline 223 & & Round & Round & Round & & Round & Round \\
\hline 224 & & Not circular & Not circular & Circular & & Circular & Circular \\
\hline 225 & & Red & Greenyellow & Blue-black & & Red & Greenyellow \\
\hline 226 & & Not uniform & Uniform & Uniform & & $\begin{array}{l}\text { Not } \\
\text { uniform }\end{array}$ & Not uniform \\
\hline $230-231$ & & Not coloured & $\begin{array}{l}\text { Slightly } \\
\text { coloured }\end{array}$ & $\begin{array}{l}\text { Slightly } \\
\text { coloured }\end{array}$ & & $\begin{array}{l}\text { Not } \\
\text { coloured }\end{array}$ & Not coloured \\
\hline 233 & & Medium & Little & Medium & & Medium & Little \\
\hline 237 & & Little flavour & Little flavour & Little aromatic & & Neutral & Neutral \\
\hline 238 & & Short & Short & Short & & Short & Short \\
\hline 239 & & Medim & Medim & Medim & & Medim & Difficult \\
\hline 241 & & Present & Present & Present & & Present & Present \\
\hline 301 & Medium & Late & Medium & Late & Early & Early & Medium \\
\hline 305 & Medium & Medium & Medium & Late & Medium & Early & Medium \\
\hline 306 & Yellow & Yellow & Yellow & Yellow & Yellow & Dark red & Red \\
\hline
\end{tabular}


Table 2. Ampelographic characteristics of grape cultivars used in this study (continued).

\begin{tabular}{|c|c|c|c|c|c|c|c|}
\hline $\begin{array}{l}\text { OIV } \\
\text { Cod } \\
\text { Number }\end{array}$ & Mahrabaşı* & Kırmızı Şam & $\begin{array}{l}\text { Kozak } \\
\text { Gemresi }\end{array}$ & $\begin{array}{l}\text { Çeşme } \\
\text { Pembesi }\end{array}$ & Malbee & Grenache & Delbele \\
\hline 001 & Half-open & Half-open & Half-open & Half-open & Half-open & Half-open & Half-open \\
\hline 003 & Very weak & Absent & Very weak & Very weak & Very weak & Very weak & Very weak \\
\hline 004 & Medium & Sparse & Sparse & Medium & Dense & Dense & Dense \\
\hline 005 & None & None & None & None & None & None & None \\
\hline 006 & Semi erect & Semi erect & Horizontal & Semi erect & Erect & Erect & Erect \\
\hline 007 & Green & G with r.s & Green & G with r.s & G with r.s & G with r.s & Green \\
\hline 008 & Green & G with r.s & G with r.s & G with r.s & Red & G with r.s & Green \\
\hline 009 & Green & G with r.s & Green & G with r.s & G with r.s & Green & Green \\
\hline 010 & Green & Green & G with r.s & Green & G with r.s & Green & Green \\
\hline 011 & None & None & None & None & Sparse & Sparse & Sparse \\
\hline 012 & None & None & None & None & None & Very sparse & Very sparse \\
\hline 013 & None & None & None & None & None & Very sparse & None \\
\hline 014 & None & None & None & None & Very sparse & Sparse & Very sparse \\
\hline 015 & Very weak & Very weak & Absent & Absent & Weak & Weak & Weak \\
\hline 016 & Dis. cont. & Dis. cont. & Dis. cont. & Dis. cont. & Sub. or cont. & Dis. cont. & Dis. cont. \\
\hline 017 & Very short & Very short & Very short & Short & Very short & Very short & Very short \\
\hline 051 & $\begin{array}{l}\text { Green with } \\
\text { b.s. }\end{array}$ & $\begin{array}{l}\text { Green with } \\
\text { b.s. }\end{array}$ & $\begin{array}{l}\text { Green with } \\
\text { b.s. }\end{array}$ & Green & $\begin{array}{l}\text { Green with } \\
\text { b.s }\end{array}$ & Green & Yellow \\
\hline 052 & None & None & None & Very weak & Weak & None & None \\
\hline 053 & Sparse & Very sparse & Very sparse & Dense & Very dense & Dense & Dense \\
\hline 054 & None & None & None & None & None & None & None \\
\hline 055 & None & None & None & None & None & None & None \\
\hline 056 & Sparse & Sparse & None & Sparse & Very sparse & Very sparse & Very sparse \\
\hline 065 & Very small & Very small & Very small & Very small & Very small & Small & Very small \\
\hline 066 & Short & Short & Short & Short & Very short & Short & Very short \\
\hline 067 & Pentagonal & Pentagonal & Wedgeshaped & Pentagonal & Pentagonal & Pentagonal & Pentagonal \\
\hline 068 & Five & Five & Five & Three & Five & Five & Five \\
\hline 069 & $\begin{array}{l}\text { Medium } \\
\text { green }\end{array}$ & Pale green & $\begin{array}{l}\text { Medium } \\
\text { green }\end{array}$ & Medium green & $\begin{array}{l}\text { Medium } \\
\text { green }\end{array}$ & Dark green & Dark green \\
\hline 070 & Absent & Absent & Absent & Absent & Absent & Absent & Absent \\
\hline 071 & Very weak & Absent & Absent & Absent & Absent & Absent & Absent \\
\hline 076 & B. s. straight & B. s. straight & B. s. straight & B. s. straight & B. s. straight & B. s. straight & B. s. straight \\
\hline 077 & Medium & Long & Medium & Very long & Medium & Long & Long \\
\hline 078 & Short & Long & Short & Long & Medium & Long & Very long \\
\hline 079 & Open & Open & Open & Wide open & Open & Open & Open \\
\hline 080 & V shape & U shape & V shape & U shape & U shape & V shape & U shape \\
\hline 081 & None & None & None & None & None & None & None \\
\hline 082 & Open & Open & Closed & L. s. overlap. & Open & Open & Open \\
\hline 083 & V shape & V shape & V shape & V shape & U shape & U shape & V shape \\
\hline 084 & None & None & None & Sparse & None & Medium & Very sparse \\
\hline 085 & None & None & None & None & None & None & None \\
\hline 086 & None & None & None & Sparse & Sprase & Medium & None \\
\hline 087 & None & None & None & Very sparse & None & None & Sparse \\
\hline 088 & Absent & Absent & Absent & Absent & Absent & Present & Absent \\
\hline 089 & Absent & Absent & Absent & Absent & Absent & Present & Absent \\
\hline 089 & Absent & Absent & Absent & Absent & Absent & Present & Absent \\
\hline 090 & None & None & None & None & Very sparse & Sparse & None \\
\hline 091 & None & None & None & None & None & None & None \\
\hline 092 & Very short & Very short & Very short & Very short & Very short & Very short & Very short \\
\hline 093 & Shorter & Shorter & Shorter & Shorter & Shorter & Shorter & Shorter \\
\hline 151 & Hermaphrodite & Hermaphrodite & Hermaphrodite & Hermaphrodite & Hermaphrodit & Hermaphrod & Hermaphrodi \\
\hline
\end{tabular}


Table 2. Ampelographic characteristics of grape cultivars used in this study (continued).

\begin{tabular}{|c|c|c|c|c|c|c|c|}
\hline $\begin{array}{l}\text { OIV } \\
\text { Cod } \\
\text { Number }\end{array}$ & Mahrabaşı* & Kırmızı Şam & $\begin{array}{l}\text { Kozak } \\
\text { Gemresi }\end{array}$ & $\begin{array}{l}\text { Çeşme } \\
\text { Pembesi }\end{array}$ & Malbee & Grenache & Delbele \\
\hline 153 & $\begin{array}{l}1.1 \text { to } 2 \\
\text { inflorescenses }\end{array}$ & $\begin{array}{l}1.1 \text { to } 2 \\
\text { inflorescenses }\end{array}$ & $\begin{array}{l}1.1 \text { to } 2 \\
\text { inflorescenses }\end{array}$ & $\begin{array}{l}1.1 \text { to } 2 \\
\text { inflorescenses }\end{array}$ & $\begin{array}{l}\text { Up ot } 1 \\
\text { inflorescence }\end{array}$ & $\begin{array}{l}1.1 \text { to } 3 \\
\text { inflorescenses }\end{array}$ & $\begin{array}{l}1.1 \text { to } 2 \\
\text { inflorescenses }\end{array}$ \\
\hline 154 & Medium & Very long & Long & Medium & Short & Medium & Short \\
\hline 203 & & Long & Short & Short & Very short & Very short & Very short \\
\hline 204 & & Very loose & Loose & Loose & Dense & Medium & Medium \\
\hline 205 & & Few & Few & Few & Very few & Very few & Very few \\
\hline 206 & & Very long & Very long & Very long & Long & Very long & Very long \\
\hline 207 & & Medium & Weak & Weak & Weak & Weak & Weak \\
\hline 221 & & Medium & Short & Long & Short & Short & Short \\
\hline 222 & & Uniform & Not uniform & Not uniform & Uniform & Uniform & Not uniform \\
\hline 223 & & Round & Round & Ovate & Round & Round & Obovate \\
\hline 224 & & Not circular & Circular & Not circular & Circular & Circular & Circular \\
\hline 225 & & Red & Red & Rose & Blue-black & Blue-black & Blue-black \\
\hline 226 & & Not uniform & Not uniform & Not uniform & Uniform & Not uniform & Not uniform \\
\hline $230-231$ & & Not coloured & $\begin{array}{l}\text { Very slightly } \\
\text { coloured }\end{array}$ & Not coloured & $\begin{array}{l}\text { Very slightly } \\
\text { coloured }\end{array}$ & Not coloured & Not coloured \\
\hline 233 & & Medium & Little & Medium & Medium & Medium & Medium \\
\hline 237 & & Little flavour & Neutral & Neutral & Neutral & Little flavour & Neutral \\
\hline 238 & & Short & Short & Short & Very short & Short & Short \\
\hline 239 & & Medim & Medim & Easy & Medim & Medim & Medim \\
\hline 241 & & Present & Present & Present & Present & Present & Present \\
\hline 301 & Medium & Medium & Early & Medium & Very early & Very early & Medium \\
\hline 305 & Early & Medium & Late & Early & Early & Early & Medium \\
\hline 306 & Yellow & Yellow & Rectviolet & Reddish & Dark red & Dark red & Yellow \\
\hline
\end{tabular}


Table 2. Ampelographic characteristics of grape cultivars used in this study (continued).

\begin{tabular}{|c|c|c|c|c|c|c|c|}
\hline $\begin{array}{l}\text { OIV Cod } \\
\text { Number }\end{array}$ & $\begin{array}{l}\text { Alicante } \\
\text { Boushet }\end{array}$ & Merlot & Foça Karası & Petit Syrah & Öküzgözü & Papaz Karası & $\begin{array}{c}\text { Cabarnet } \\
\text { Sauvignon* }\end{array}$ \\
\hline 001 & Half-open & Half-open & Half-open & Half-open & Half-open & Half-open & Half-open \\
\hline 003 & Weak & Very weak & Very weak & Very weak & Very weak & Very weak & Medium \\
\hline 004 & Dense & Dense & Seyrek & Dense & Medium & Dense & Dense \\
\hline 005 & None & None & None & None & None & None & None \\
\hline 006 & Semi erect & Erect & Semi erect & Semi erect & Horizontal & Semi erect & Semi erect \\
\hline 007 & G with r.s & $\mathrm{G}$ with r.s & Green & G with r.s & G with r.s & G with r.s & G with r.s \\
\hline 008 & G with r.s & G with r.s & G with r.s & Green & G with r.s & G with r.s & G with r.s \\
\hline 009 & G with r.s & G with r.s & G with r.s & G with r.s & G with r.s & G with r.s & G with r.s \\
\hline 010 & G with r.s & G with r.s & Green & G with r.s & G with r.s & Green & Red \\
\hline 011 & None & None & None & None & None & Sparse & None \\
\hline 012 & Very sparse & None & None & None & None & None & None \\
\hline 013 & None & Very sparse & None & None & None & Sparse & Sparse \\
\hline 014 & Very sparse & Very sparse & None & None & None & Sparse & Sparse \\
\hline 015 & Very weak & Very weak & Absent & Absent & Absent & Absent & Very weak \\
\hline 016 & Dis. cont. & Dis. cont. & Dis. cont. & Dis. cont. & Dis. cont & Dis. cont. & Dis. cont. \\
\hline 017 & Very short & Short & Very short & Very short & Very short & Very short & Short \\
\hline 051 & $\begin{array}{l}\text { Green with } \\
\text { b.s. }\end{array}$ & Yellow & $\begin{array}{l}\text { Green with } \\
\text { b.s. }\end{array}$ & Green with b.s. & $\begin{array}{l}\text { Green with } \\
\text { b.s. }\end{array}$ & Yellow & $\begin{array}{l}\text { Green with } \\
\text { b.s. }\end{array}$ \\
\hline 052 & None & None & None & None & Weak & None & None \\
\hline 053 & Very dense & Very dense & Sparse & Very dense & Very sparse & Very dense & Dense \\
\hline 054 & None & None & None & None & None & None & None \\
\hline 055 & None & None & None & None & Very sparse & Medium & None \\
\hline 056 & Sparse & Sparse & Sparse & Very sparse & None & Very sparse & Very sparse \\
\hline 065 & Very small & Very smal & Very small & Very small & Very small & Very small & Very small \\
\hline 066 & Short & Short & Very short & Very short & Short & Short & Very short \\
\hline 067 & Wedgeshaped & Pentagonal & Pentagonal & Pentagonal & Pentagonal & Pentagonal & Pentagonal \\
\hline 068 & Five & Five & Five & Five & Five & Five & Five \\
\hline 069 & Dark green & Dark green & Medium green & Medium green & Medium green & Dark green & Dark green \\
\hline 070 & Medium & Absent & Absent & Absent & Very weak & Absent & Absent \\
\hline 071 & Weak & Absent & Absent & Absent & Very weak & Absent & Absent \\
\hline 076 & B. s. straight & B. s. straight & B. s.straight & B. s. straight & O. S. c, o. S. C. & B. s. straight & B. s. straight \\
\hline 077 & Long & Long & Medium & Medium & Short & Long & Long \\
\hline 078 & Medium & Long & Long & Medium & Long & Long & Long \\
\hline 079 & Open & Open & Open & Open & Open & Open & Open \\
\hline 080 & V shape & U shape & V shape & V shape & V shape & V shape & U shape \\
\hline 081 & None & None & None & None & None & None & None \\
\hline 082 & Open & Open & Open & Open & Open & Open & L. s. overlap. \\
\hline 083 & V shape & U shape & V shape & V shape & U shape & U shape & U shape \\
\hline 084 & Dense & Sparse & None & Medium & None & Dense & Sparse \\
\hline 085 & None & None & None & None & None & None & None \\
\hline 086 & Sprase & Very sprase & None & Medium & None & Sparse & None \\
\hline 087 & None & None & None & None & None & None & Very sparse \\
\hline 088 & Absent & Present & Absent & Absent & Absent & Present & Absent \\
\hline 089 & Absent & Absent & Absent & Absent & Absent & Absent & Absent \\
\hline 089 & Absent & Absent & Absent & Absent & Absent & Absent & Absent \\
\hline 090 & None & None & None & None & None & None & None \\
\hline 091 & Very sparse & None & None & None & None & None & None \\
\hline 092 & Very short & Very short & Very short & Very short & Very short & Very short & Very short \\
\hline 093 & Shorter & Shorter & Shorter & Shorter & Shorter & Shorter & Shorter \\
\hline 151 & Hermaphrodite & Hermaphrodite & Hermaphrodite & Hermaphrodite & Hermaphrodite & Hermaphrodite & Hermaphrodite \\
\hline 153 & $\begin{array}{l}1.1 \text { to } 2 \\
\text { inflorescenses }\end{array}$ & $\begin{array}{l}\text { Up to } 1 \\
\text { inflorescence }\end{array}$ & $\begin{array}{l}1.1 \text { to } 2 \\
\text { inflorescenses }\end{array}$ & $\begin{array}{l}1.1 \text { to } 2 \\
\text { inflorescense }\end{array}$ & $\begin{array}{l}1.1 \text { to } 2 \\
\text { inflorescenses }\end{array}$ & $\begin{array}{l}1.1 \text { to } 2 \\
\text { inflorescenses }\end{array}$ & $\begin{array}{l}\text { Up to } 1 \\
\text { inflorescence }\end{array}$ \\
\hline
\end{tabular}


Table 2. Ampelographic characteristics of grape cultivars used in this study (continued).

\begin{tabular}{|c|c|c|c|c|c|c|c|}
\hline $\begin{array}{l}\text { OIV } \\
\text { Cod } \\
\text { Number }\end{array}$ & $\begin{array}{l}\text { Alicante } \\
\text { Boushet }\end{array}$ & Merlot & Foça Karası & Petit Syrah & Öküzgözü & Papaz Karası & $\begin{array}{c}\text { Cabarnet } \\
\text { Sauvignon* }\end{array}$ \\
\hline 153 & $\begin{array}{l}1.1 \text { to } 2 \\
\text { inflorescenses }\end{array}$ & $\begin{array}{l}\text { Up to } 1 \\
\text { inflorescence }\end{array}$ & $\begin{array}{l}1.1 \text { to } 2 \\
\text { inflorescenses }\end{array}$ & $\begin{array}{l}1.1 \text { to } 2 \\
\text { inflorescenses }\end{array}$ & $\begin{array}{l}1.1 \text { to } 2 \\
\text { inflorescenses }\end{array}$ & $\begin{array}{l}1.1 \text { to } 2 \\
\text { inflorescenses }\end{array}$ & $\begin{array}{l}\text { Up to } 1 \\
\text { inflorescence }\end{array}$ \\
\hline 154 & Long & Medium & Medium & Medium & Medium & Medium & Medium \\
\hline 203 & Very short & Very short & Very short & & Short & Medium & \\
\hline 204 & Very dense & Very dense & Very dense & & Loose & Very dense & \\
\hline 205 & Very few & Medium & Medium & & Very few & Medium & \\
\hline 206 & Very long & Short & & Short & Very short & Very long & \\
\hline 207 & Weak & Weak & Weak & Medium & Medium & Weak & \\
\hline 221 & Short & Short & & Short & Medium & Short & \\
\hline 222 & Not uniform & Uniform & & Uniform & Not uniform & Not uniform & \\
\hline 223 & Slightly flat & Round & & Round & Slightly flat & Round & \\
\hline 224 & Not circular & Circular & & Circular & Circular & Circular & \\
\hline 225 & Blue-black & Blue-black & & Blue-black & Rose & Red & \\
\hline 226 & Not uniform & Uniform & & Not uniform & Uniform & Uniform & \\
\hline $230-231$ & $\begin{array}{l}\text { Very strong } \\
\text { coloured }\end{array}$ & Very slightly & & Not coloured & Very slightly & Not coloured & \\
\hline 233 & Medium & Medium & & Medium & Medium & Medium & \\
\hline 237 & Little flavour & $\begin{array}{l}\text { Little } \\
\text { aromatic }\end{array}$ & & Neutral & Little flavour & Little flavour & \\
\hline 238 & Short & Very short & & Short & Short & Short & \\
\hline 239 & Medium & Medium & & Medium & Medium & Easy & \\
\hline 241 & Present & Present & & Present & Present & Absent & \\
\hline 301 & Medium & Çok erken & Medium & Very early & Medium & Medium & Early \\
\hline 305 & Early & Early & Medium & Very early & Medium & Medium & Early \\
\hline 306 & Rectviolet & Red & Yellow & Red-violet & Yellow & Red & Rectviolet \\
\hline
\end{tabular}


Table 2. Ampelographic characteristics of grape cultivars used in this study (continued).

\begin{tabular}{|c|c|c|c|c|c|c|c|}
\hline $\begin{array}{l}\text { OIV } \\
\text { Cod } \\
\text { Number }\end{array}$ & Cabarnet Franc ${ }^{*}$ & Cinsaut & $\begin{array}{l}\text { Grenache } \\
\text { Noir }^{*}\end{array}$ & Colombard & Harsleleh & Semillion & Abiguş $^{*}$ \\
\hline 001 & Half-open & Half-open & Half-open & Half-open & Half-open & Half-open & Half-open \\
\hline 003 & Medium & Weak & Very weak & Absent & Very weak & Weak & Absent \\
\hline 004 & Medium & Dense & Sparse & Dense & Dense & Medium & Very sparse \\
\hline 005 & None & None & None & None & None & None & None \\
\hline 006 & Erect & Semi erect & Erect & Semi erect & Semi erect & Semi erect & \\
\hline 007 & G with r.s & Green & Green & G with r.s & G with r.s & Green & \\
\hline 008 & Red & Green & Green & G with r.s & G with r.s & G with r.s & \\
\hline 009 & Red & G with r.s & Green & G with r.s & G with r.s & Green & \\
\hline 010 & Red & G with r.s & Green & G with r.s & G with r.s & G with r.s & \\
\hline 011 & None & None & None & None & None & None & \\
\hline 012 & Very sparse & None & None & None & None & None & \\
\hline 013 & None & None & None & None & None & None & \\
\hline 014 & Very sparse & None & None & Sparse & Very sparse & None & \\
\hline 015 & Very weak & Absent & Absent & Medium & Absent & Very weak & \\
\hline 016 & Dis. cont. & Dis. cont. & Dis. cont. & Dis. cont. & Dis. cont. & Dis. cont. & \\
\hline 017 & Very short & Very short & Very short & Very short & Very short & Very short & \\
\hline 051 & Reddish & Reddish & Green & Green & Yellow & $\begin{array}{l}\text { Green with } \\
\text { b.s. }\end{array}$ & Green \\
\hline 052 & Weak & None & None & None & None & Very weak & \\
\hline 053 & Very dense & Dense & Very sparse & Very dense & Very dense & Dense & Very sparse \\
\hline 054 & None & None & None & None & None & None & \\
\hline 055 & None & Very sparse & None & Sparse & Medium & Sparse & \\
\hline 056 & Very sparse & Spare & Sparse & None & Sparse & Very sparse & None \\
\hline 065 & Very small & Very small & Very small & Very small & Very small & Very small & \\
\hline 066 & Very short & Very short & Very short & Short & Short & Very short & \\
\hline 067 & Pentagonal & Pentagonal & Pentagonal & Pentagonal & Cordate & Pentagonal & \\
\hline 068 & Five & Five & Five & Five & Five & Five & \\
\hline 069 & Dark green & Medium green & Pale green & Dark green & Medium green & Açık yeşil & \\
\hline 070 & Very weak & Absent & Absent & Absent & Absent & Absent & \\
\hline 071 & Very weak & Absent & Absent & Absent & Absent & Absent & \\
\hline 076 & B. s. straight & B. s. straight & B. s. straight & B. s. straight & B. s. straight & B. s. straight & \\
\hline 077 & Medium & Long & Medium & Medium & Medium & Long & \\
\hline 078 & Long & Short & Short & Short & Short & Medium & \\
\hline 079 & Open & Open & Open & Open & Open & Wide open & \\
\hline 080 & U shape & V shape & V shape & V shape & U shape & U shape & \\
\hline 081 & None & None & None & None & None & None & \\
\hline 082 & Open & Open & L. s. overlap. & Open & Open & L. s. overlap. & \\
\hline 083 & U shape & V shape & V shape & U shape & V shape & U shape & \\
\hline 084 & Sprase & Very sprase & None & Medium & Medium & Very sprase & \\
\hline 085 & None & Very weak & None & Weak & None & None & \\
\hline 086 & None & None & Very sparse & None & Sprase & Very sprase & \\
\hline 087 & None & Very sparse & None & Sparse & None & None & \\
\hline 088 & Present & Absent & Absent & Absent & Absent & Absent & \\
\hline 089 & Absent & Absent & Absent & Absent & Absent & Absent & \\
\hline 090 & None & Very sparse & None & None & None & None & \\
\hline 091 & Very sparse & None & None & None & Sparse & Very dense & \\
\hline 092 & Very short & Very short & Very short & Very shott & Very short & Very short & \\
\hline 093 & Shorter & Shorter & Shorter & Shorter & Shorter & Shorter & \\
\hline 151 & Hermaphrodite & Hermaphrodite & Hermaphrodite & Hermaphrodite & Hermaphrodite & Hermaphrodite & \\
\hline 153 & $\begin{array}{l}1.1 \text { to } 2 \\
\text { inflorescenses }\end{array}$ & $\begin{array}{l}1.1 \text { to } 2 \\
\text { inflorescenses }\end{array}$ & $\begin{array}{l}1.1 \text { to } 2 \\
\text { inflorescenses }\end{array}$ & \begin{tabular}{|l|}
1.1 to 2 \\
inflorescenses
\end{tabular} & $\begin{array}{l}1.1 \text { to } 2 \\
\text { inflorescenses }\end{array}$ & \begin{tabular}{|l|l|}
1.1 to 2 \\
inflorescenses
\end{tabular} & \\
\hline
\end{tabular}


Table 2. Ampelographic characteristics of grape cultivars used in this study (continued).

\begin{tabular}{|c|c|c|c|c|c|c|c|}
\hline $\begin{array}{l}\text { OIV } \\
\text { Cod } \\
\text { Number }\end{array}$ & Cabarnet Franc* & Cinsaut & $\begin{array}{c}\text { Grenache } \\
\text { Noir* }\end{array}$ & Colombard & Harsleleh & Semillion & Abiguş* \\
\hline 154 & Short & Medium & Medium & Short & Medium & Medium & \\
\hline 203 & & Very short & & Very short & Very short & Very short & \\
\hline 204 & & Dense & & Medium & Loose & Dense & \\
\hline 205 & & Very few & & Few & Medium & Few & \\
\hline 206 & & Long & & Long & Long & Long & \\
\hline 207 & & Medium & & Weak & Weak & Weak & \\
\hline 221 & & Short & & Short & Short & Short & \\
\hline 222 & & Uniform & & Not uniform & Not uniform & Uniform & \\
\hline 223 & & Long elliptic & & Obtuseovate & Round & Slightly flat & \\
\hline 224 & & Circular & & Not circular & Circular & Not circular & \\
\hline 225 & & Blue-black & & Greenyellow & Rose & Rose & \\
\hline 226 & & Not uniform & & Not uniform & Not uniform & Not uniform & \\
\hline $230-231$ & & Not coloured & & Not coloured & Not coloured & Not coloured & \\
\hline 233 & & Medium & & Medium & Medium & Medium & \\
\hline 237 & & Neutral & & Neutral & Little aromatic & Neutral & \\
\hline 238 & & Short & & Short & Short & Short & \\
\hline 239 & & Difficult & & Easy & Easy & Medium & \\
\hline 241 & & Present & & Present & Present & Absent & \\
\hline 301 & Early & Very early & Late & Very early & Medium & Early & \\
\hline 305 & Early & Very early & Medium & Early & Medium & Medium & \\
\hline 306 & Dark red & Red-violet & Yellow & Yellow & Yellow & Yellow & \\
\hline
\end{tabular}

G with r.s: Green with red stripes; Dis. cont.: Discontinuous (2 or less); Sub. or cont.: Subcontinuous or continuous (3 or more); Green with b.s.: Green with bronze spots; B. s. straight: Both sides straight; O. s. c, o. s. c.: One side concave, one side convex; L. s. overlap.: Lobes slightly overlapping.

Tarsus Pembesi*; Cabernet Franc*; Abiguş*; Cabernet Sauvignon*; Beyaz Şam*;

Pembe Gemre*; Mahrabaşı*; Granache Noir*: Grape varieties that could not be observed in their maturity due to lack of sufficient number of clusters that could be analyzed. 


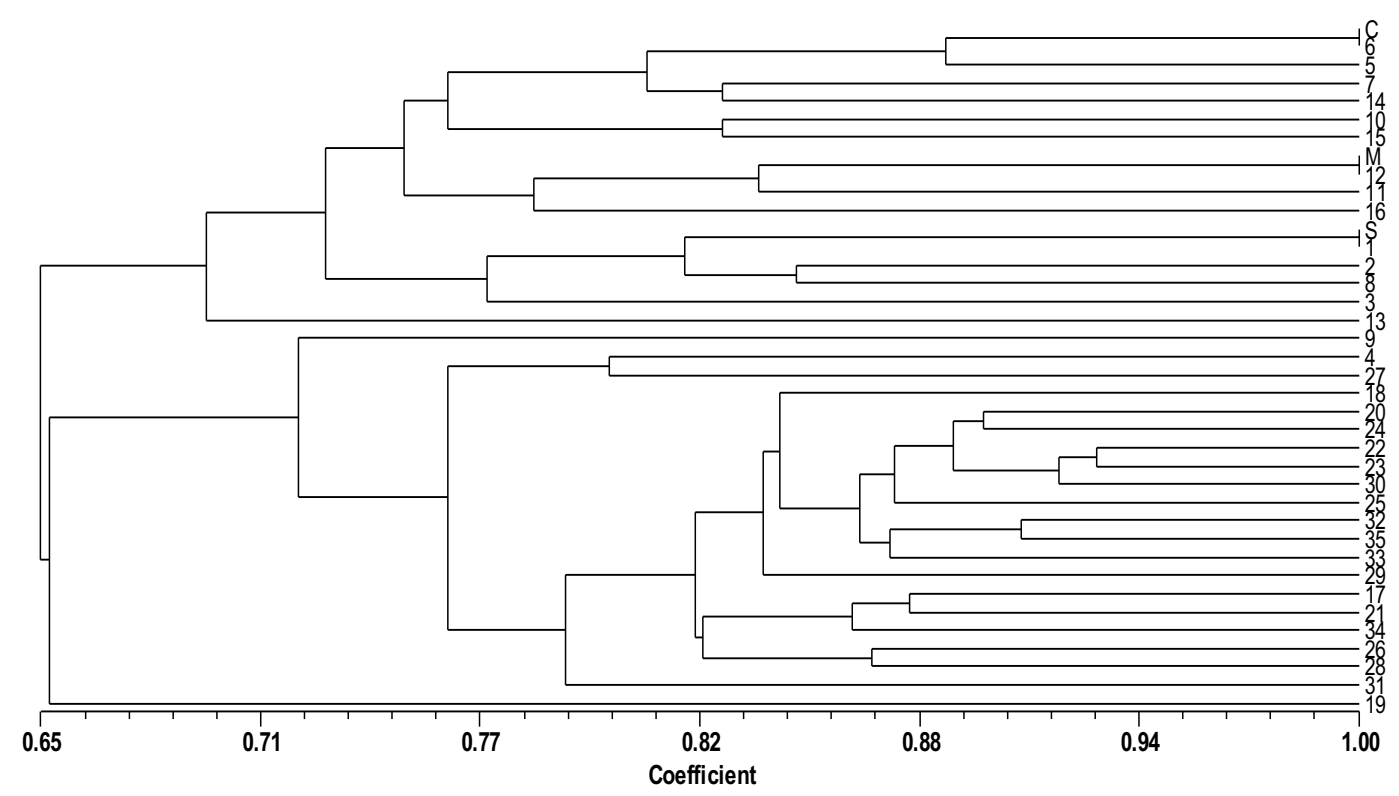

Figure 1. Dendrogram showing the relationships of 38 grapevine cultivars used in this study based on UPGMA cluster analysis of 73 features in "Descriptors for Grape"

C: Cabernet Sauvignon, M: Merlot, S: Sémillon, 1: Sémillon, 2: Marsleleh, 3: Conlonbart, 4: Grenache Noir, 5: Cinsaut, 6: Cabernet Franc, 7: Cabernet Sauvignon, 8: Papazkaras1, 9: Öküzgözü, 10: Petit Syrah, 11: Foça Karas1, 12: Merlot, 13: Alicante Boushet, 14: Delbele, 15: Grenache, 16: Malbee, 17: Çeşme Pembesi, 18: Kozak Gemresi 19: Abiguş, 20: Kırmızı Şam, 21: Mahrabaşı, 22: Yuvarlak Razakı, 23: Siyah Gemre, 24: Pembe Gemre, 25: Cardinal, 26: Beyaz Şam,27: Italia, 28: Ohannes, 29: Pek Üzümü, 30: Şika, 31: Müşküle, 32: Moiseylative, 33: Buca Razakısı, 34: Tarsus Pembesi, 35: Hafizali

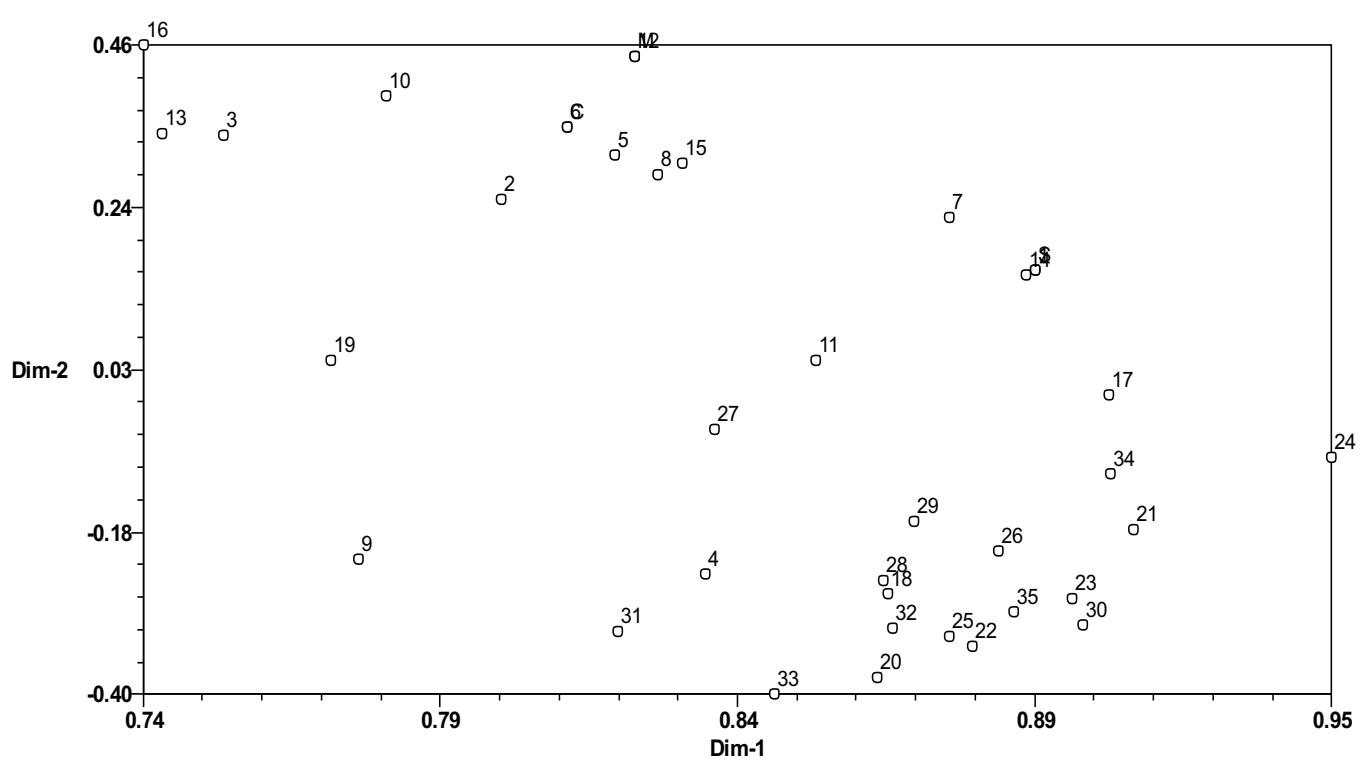

Figure 2. Principal component analyses of 38 grapevine cultivars used in this study based on 73 ampelographic characteristics

C: Cabernet Sauvignon, M: Merlot, S: Sémillon, 1: Sémillon, 2: Marsleleh, 3: Conlonbart, 4: Grenache Noir, 5:

Cinsaut, 6: Cabernet Franc, 7: Cabernet Sauvignon, 8: Papazkaras1, 9: Öküzgözü, 10: Petit Syrah, 11: Foça Karası, 12: Merlot, 13: Alicante Boushet, 14: Delbele, 15: Grenache, 16: Malbee, 17: Çeşme Pembesi, 18: Kozak Gemresi 19:

Abiguş, 20: Kırmızı Şam, 21: Mahrabaş1, 22: Yuvarlak Razak1, 23: Siyah Gemre, 24: Pembe Gemre, 25: Cardinal, 26: Beyaz Şam,27: Italia, 28: Ohannes, 29: Pek Üzümü, 30: Şika, 31: Müşküle, 32: Moiseylative, 33: Buca Razakıs1, 34: Tarsus Pembesi, 35: Hafizali 